\title{
Pancytopenia and Kaposi sarcoma indicators of HIV infection diagnosis: A case report
}

\author{
Monica Padurariu-Covit ${ }^{1,2}$, Gabriela Lupasteanu ${ }^{3,4}$, Manuela Arbune ${ }^{1,3}$ \\ ${ }^{1}$ Faculty of Medicine and Pharmacy, "Dunarea de Jos" University, Galati, Romania \\ 2"Sf. Apostol Andrei" Emergency Districtual Clinic Hospital, Galati, Romania \\ ${ }^{3}$ Infectious Diseases Clinic Hospital, Galati \\ ${ }^{4}$ Doctoral School, "Ovidius" University, Constanta, Romania
}

\begin{abstract}
During HIV infection, hematological manifestations are frequently associated with various pathogenic mechanisms, which require a multidisciplinary approach. We present the case of a 57-year-old patient with pancytopenia associated with Kaposi's sarcoma, as indicative manifestations of the acquired immunodeficiency syndrome. The late diagnosis of HIV infection, in the stage of severe immunosuppression, the association of opportunistic neoplasia, delayed oncological therapy and the development of immune reconstruction syndrome after starting antiretroviral therapy have contributed to the severe evolution and death of the patient.
\end{abstract}

Keywords: HIV infection, pancytopenia, Kaposi's sarcoma, IRIS

\section{INTRODUCTION}

Pancytopenia is a syndrome often found in hematological clinical practice, defined by the concomitant decrease of the three hematopoietic lines, characterized by hemoglobin values $<10 \mathrm{~g} / \mathrm{dl}$, leukocytes $<4,000 / \mathrm{mmc}$ and platelets $<150,000 / \mathrm{mmc}$ (1).

Pancytopenia is not a disease by itself, but can suggest the first sign of several pathologies, with various etiologies, which are classified into the following groups: decreased bone marrow function (spinal cord aplasia, spinal cord infiltration), inefficient erythropoiesis (megaloblastic anemia) or increased peripheral destruction (2). Human immunodeficiency virus (HIV) infection is often associated with pancytopenia, which can occur through multiple mechanisms, especially induced by the direct cytopathic effect of HIV, opportunistic infections, immune mechanisms, neoplasms, drug toxicity (3). Frequency and severity of HIV-associated hemato- logical manifestations are higher in advanced stages of immunosuppression (AIDS), characterized by high viral replication and low levels of T-CD4 lymphocytes. Chronic inflammatory response, accompanied by the release of cytokines that can change all lines of hematopoiesis and increase cytopenia by immune mechanisms (4).

Hematological disorders can be inaugural to the diagnosis of HIV, even in the absence of clinical symptoms.

\section{CASE PRESENTATION}

We present the case of a 57-year-old patient, retired, from urban areas, who presented to the hematology service for the suspicion of a lymphoproliferative syndrome, in the context of lymphadenopathy, accompanied by fever and weight loss $\sim 10 \%$ of body weight, with a progressive evolution in the last 3 months. The late presentation to the doctor was motivated by the pandemic 
context of COVID-19 and the fear of contacting the disease. The medical history mentions a natural birth, two abortions, duodenal ulcer, osteoporosis, tachyarrhythmia and a persistent biological syndrome of 3 years, apparently unexplained. She denies smoking, drinking alcohol or recreational substances and has been living alone for over 10 years, being divorced.

The clinical exam revealed: moderately influenced general condition, conscious, with depressive mood, afebrile, BP = 130/80 mmHg, HR = $100 \mathrm{bpm}$, $\mathrm{FR}=16$ breaths $/ \mathrm{min}, \mathrm{SO} 2=97 \%$ in the ambient air; bilateral enlargement of the lymph nodes, of 2-3 cm diameter, hard consistency, fixed to the underlying tissues, located submandibular, laterocervical, supraclavicular, axillary and inguinal; slightly infiltrated read-purple skin lesions, with dimensions between 1 and $3 \mathrm{~cm}$, located at the left oral commissure, upper chest, right breast and submammary region; tonsillar hypertrophy, diffuse purplish erythema on the hard palate with infiltrative appearance, dentures and candidiasis were found on the oral examination (see figure 1).

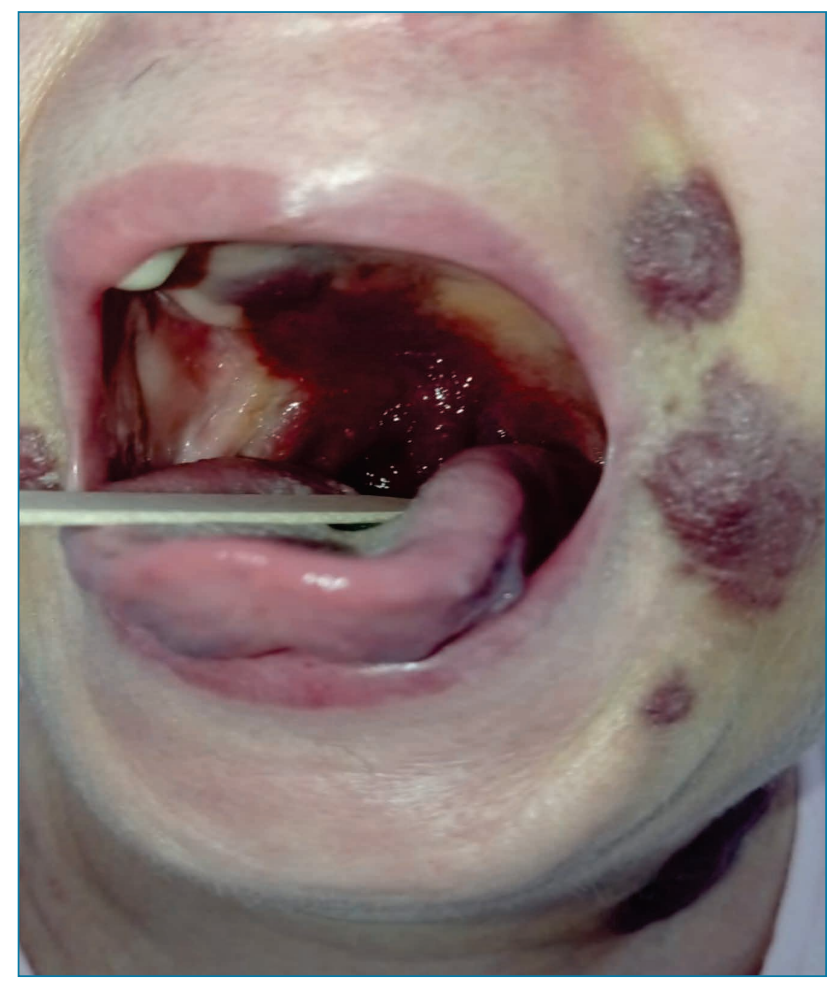

FIGURE 1. Cutaneous and oral Kaposi's sarcoma in a patient with HIV immunodepression

Laboratory tests revealed pancytopenia, severe inflammatory syndrome, hypoalbuminemia (Table 1). Tumor markers CA19.9, CA125, CA15.3, ACE were within safe limits. The medullogram exam, with discrete growth of plasma cells, slight suppression on the erythrocyte and granulocyte lines, usually implies a persistent inflammatory syndrome.
Contrast-enhanced cervico-thoraco-abdominopelvic CT-scan confirms clinically described peripheral lymphadenopathy, the association of mediastinal and retroperitoneal lymphadenopathy, and the presence of a $25 \mathrm{~mm}$ liquid blade in the pouch of Douglas.

The macroscopic appearance of the skin lesions was suggestive of Kaposi's sarcoma, that is frequently related to severe immunosuppression human immunodeficiency virus (HIV). The infection was confirmed by ELISA and Western Blot tests. The biopsy of the right supraclavicular lymph node was performed. The histopathological findings, correlated with immunohistochemical tests, were matching with Kaposi's sarcoma of the lymph node, HHV8 positive in vascular proliferation (Table 1 ).

The first specific evaluation for HIV infection indicates high viral replication, with HIV-RNA = 368,000 copies $/ \mathrm{ml}$ and severe immunosuppression of LT-CD4 $=42 / \mathrm{mmc}$, classifying as C3 stage, according to $\mathrm{CDC}(5,6)$. Markers for HBV, HCV, lues were negative. She received antifungal treatment for Candidiasis (Fluconazole), prophylaxis of pneumocystosis and atypical mycobacteria (Cotrimoxazole, Clarithromycin) and antiretroviral treatment with Genvoya (Elvitegravir / Cobicistat / Emtricitabine / Tenofovir Alafenamide). The hematological evolution seemed favorable after 6 weeks, due to the improvement of the count blood cells, the increase of L-CD4 to $192 / \mathrm{mmc}$, along with the significant decrease of the viral replication. However, the general condition was worsening, with fever, extensive skin and oral mucosal lesions, difficulty in swallowing and breathing. This paradoxical evolution fits within the definition of inflammatory immune reconstruction syndrome (IRIS), with clinical outcome improved after corticosteroid therapy, in the next 2 weeks. The serial CT imaging revealed the bilateral extension of the enlarged lymph nodes, in the upper and middle abdomen, retroperitoneal, iliac, mediastinal and cardio-phrenic, and the appearance of right pleurisy and iodophilic infiltrates in the soft parts of the epicranial, cervical, parotid, mouth and hypopharynx. Examination of pleural fluid and pneumology evaluation ruled out tuberculosis. Further, febrile episodes reappeared, with predominantly nocturnal sweating, weight loss and progressive clinical decline, dying after $16^{\text {th }}$ weeks from HIV diagnosis.

\section{DISCUSSION}

Kaposi's sarcoma (KS) is an angioproliferative tumor, derived from endothelial cells, with multifactorial etiology, associated with human herpesvirus 8 (HHV-8) infection (7). Depending on the circumstances in which KS is expressed, there are 
TABLE 1. Biological evolution after diagnosis of HIV to death (WO-W16)

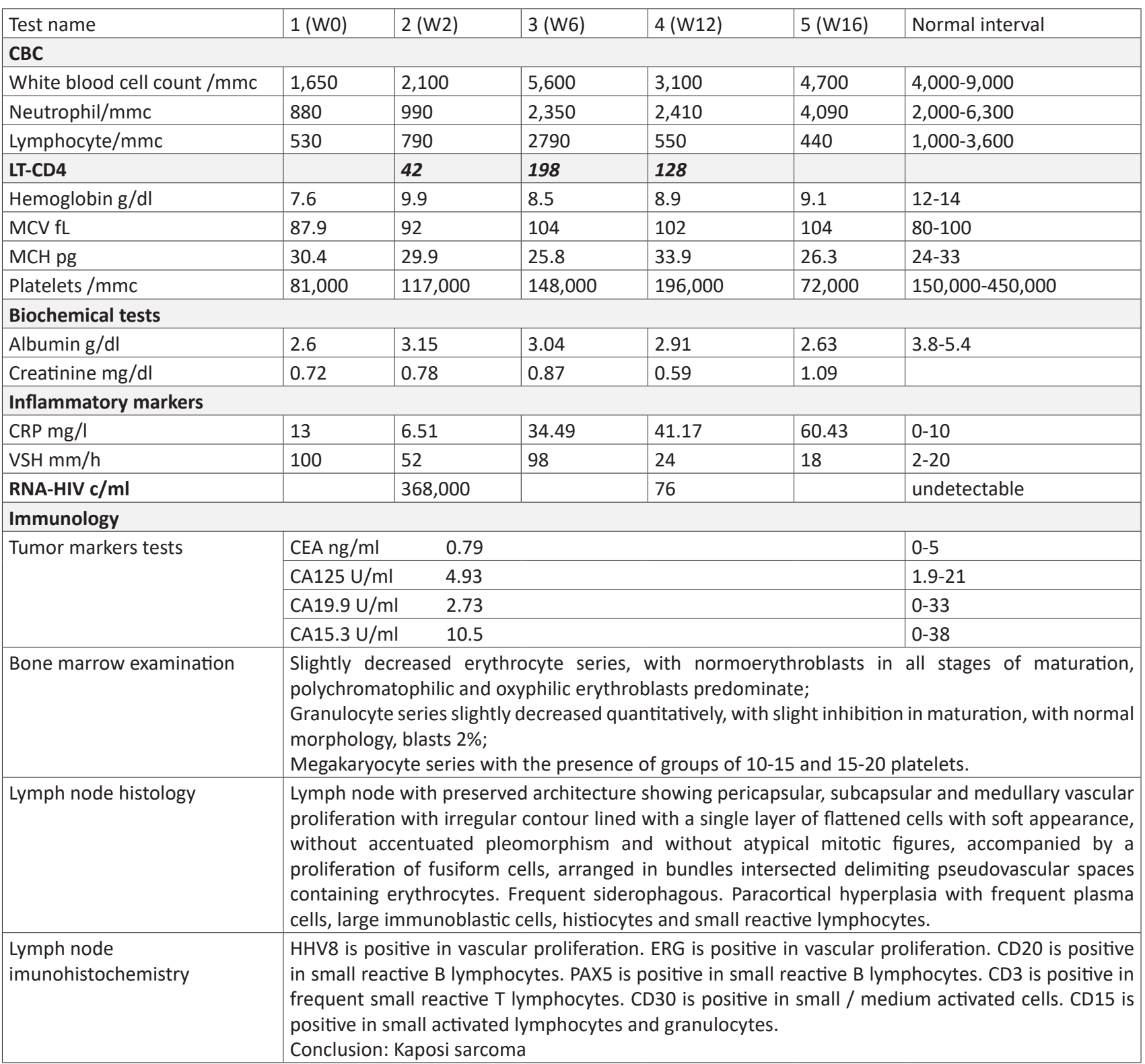

recognized four subtypes: classic, occurring in the elderly; endemic, described in indigenous Africans in the sub-Saharan region, iatrogenic, developed after immunosuppressive therapy, especially after kidney transplantation, epidemic, associated with acquired immunodeficiency syndrome (AIDS) $(8,9)$.

Epidemic KS is the most common AIDS-related cancer that occurs in the United States (6). Although KS was more than 20,000 times more common in people with AIDS than the general population, the incidence has dropped significantly after highly active antiretroviral therapy (ART) become largely accessible. Nowadays, KS is rare reported but with high mortality $(10,11)$. The KS frequency is $2-3$ times higher in men than in women (12). The spectrum of clinical manifestations includes: forms limited to red-purple or brown skin lesions, oral mucosal lesions, lymphadenopathy, lymphedema and / or vis- ceral lesions with various locations, especially pulmonary and gastrointestinal (10-12).

A particular situation for KS in patients with severe acquired immunosuppression is the development of inflammatory reconstruction immune syndrome (IRIS) after the initiation of ART, in the first weeks after the start of therapy (13). This syndrome is characterized by a paradoxical inflammatory response, as a result of decreased viral load and restoration of L-CD4 immunity, manifested in the first 3 months after initiating ART, by exposing a latent infection or by aggravating the symptoms of an opportunistic infection, already diagnosed and in treatment $(12,13)$.

The incidence of IRIS varies for different cohorts, being more commonly presented as tuberculosis, abscesses and folliculitis, varicella zoster virus infections, herpes simplex, cryptococcal 
TABLE 2. Particularities of the case

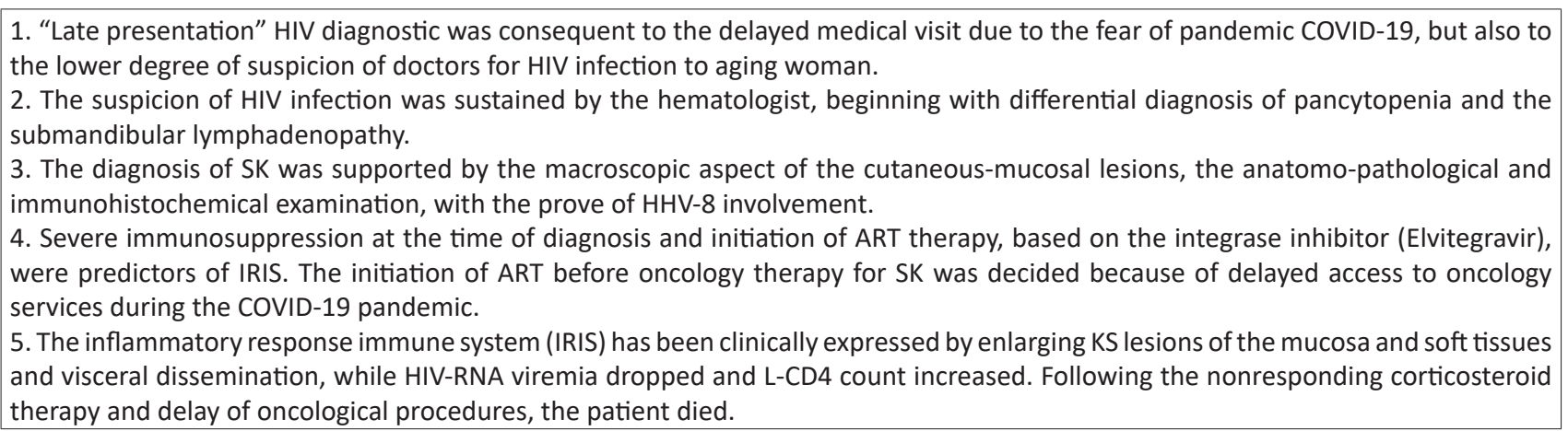

meningitis, molluscum contagiosum or Kaposi's sarcoma, which contribute to increased mortality from these diseases $(14,15)$.

The most common system for scoring the prognostic risk of KS belongs to the ACTG Oncology Committee (AIDS Clinical Trials Group), which considers the distribution of the tumor, the number of LCD4, the presence of opportunistic infections and the dependence scoring. Biomarkers with prognostic significance for survival prediction are L-CD4 number, LT-CD4 / LT-CD8 ratio, hematocrit, $\beta 2$-microglobulin, neopterin $(16,17)$.

The pathogenesis of SK in IRIS was explained by the dysfunction of the host inflammatory response, which involves the activation of HHV8 antigens (18). Decreased viral replication and increased LCD4 increase the release of inflammatory cytokines, which promote HHV8 gene expression, viral antigen production, and reversal of the Th2 (CD4) / Th1 (CD8) immune response, amplifying the production of inflammatory and LT-cytotoxic cytokines. Aberrant signals of excessive inflammation promote endothelial cell angiogenesis, contributing to the manifestations of KS (19).

Therapy of cutaneous forms of KS could be limited to highly active antiretroviral therapy. Other options for the treatment of symptomatic forms of KS are surgery by local excision, radiotherapy, cryo- therapy, laser therapy or topical retinoids ointments. Chemotherapy is recommended for visceral, oral or lymph node involvement. First-line liposomal doxorubicin, or second-line paclitaxel regimens are recommended every two or three weeks. Pomalidomide, Gemcitabine, Lenalidomide Vinorelbine could be used following the failure on previous two therapeutic lines $(16,17,18,20)$.

We summarized in table 2 the particularities of our case.

\section{CONCLUSIONS}

Pancytopenia could be an indication of acquired immunosuppression, irrespective of age and differential diagnosis requires systematic HIV testing recommendation by hematological protocols.

The extension of Kaposi's sarcoma after initiating antiretroviral therapy in advanced HIV immunosuppression is a severe manifestation of the inflammatory syndrome of immune reconstruction, with potentially fatal evolution.

The difficulties of the multidisciplinary approach of the patient with HIV infection and opportunistic neoplastic disease, the delayed access to diagnostic services and onco-hematological therapy, are amplified in the pandemic context of COVID-19, proving the vulnerability of these people.

Conflict of interest: none declared Financial support: none declared

\section{REFERENCES}

1. Hayat AS, Khan AH, Baloch GH, et al. Pancytopenia; study for clinical features and etiological pattern at tertiary care settings in Abbottabad. Professional Med J. 2014;21(1): 60-65.

2. Rehmani TH, Arif M, Haider $S$, et al. Spectrum of pancytopenia, a tertiary care experience. Professional Med J. 2016;23(5):620-626.

3. Santiago-Rodríguez EJ, Mayor AM, Fernández-Santos DM, HunterMellado RF. Profile of HIV-Infected Hispanics with Pancytopenia. Int J Environ Res Public Health. 2015 Dec 22;13(1):ijerph13010038.

4. Servais J, Nkoghe D, Schmit JC, Arendt V, et al. HIV-associated hematologic disorders are correlated with plasma viral load and improve under highly active antiretroviral therapy. J Acquir Immune Defic Syndr. 2001 Nov 1;28(3):221-5.

5. European Centre for Disease Prevention and Control. WHO Regional Office for Europe. HIV/AIDS surveillance in Europe 2020 - 2019 data. Copenhagen: WHO Regional Office for Europe; 2020.

6. CDC - Centers for Disease Control and Prevention 1992a. Revised classification system for HIV infection and expanded surveillance case definition for AIDS among adolescents and adults. MMWR. 41(RR-17):1-19.

7. Mesri EA, Cesarman E, Boshoff C. Kaposi's sarcoma and its associated herpesvirus. Nat Rev Cancer. 2010 Oct;10(10):707-19.

8. Lima CT, Araújo PSR, Teixeira HM, Santos JBD, Silveira VMD. Clinical and laboratory characteristics, staging, and outcomes of individuals 
with AIDS-associated Kaposi's sarcoma at an university hospital. An Bras Dermatol. 2017;92(2):172-176.

9. Cesarman E, Damania B, Krown SE, Martin J, Bower M, Whitby D. Kaposi sarcoma. Nat Rev Dis Primers. 2019;5(1):9.

10. Caimoni $M$, Raimondo $M$, Regine $V$, et al. Late presenters among persons with a new HIV diagnosis in Italy, 2010-2011. BMC Public Health. 2013;281(13):1471-2558.

11. Borghi V, Girardi E, Bellelli S, et al. Late Presenters in an HIV Surveillance System in Italy During the Period 1992-2006. Journal Acquir Immune Defic Syndr. 2008;49:282-286.

12. El Amari EB, Toutous-Trellu L, Gayet-Ageron A, Baumann M, et al.; Swiss HIV Cohort Study. Predicting the evolution of Kaposi sarcoma, in the highly active antiretroviral therapy era. AIDS. 2008 May 31;22(9):1019-28.

13. Murdoch DM, Venter WD, Van Rie A, Feldman C. Immune reconstitution inflammatory syndrome (IRIS): review of common infectious manifestations and treatment options. AIDS Res Ther. 2007;4:9.

14. Gopal R, Rapaka RR, Kolls JK. Immune reconstitution inflammatory syndrome associated with pulmonary pathogens. Eur Respir Rev. 2017;26(143):160042.

15. Jipa R, Săndulescu $O$, Manea $E$, et al. Kaposi Sarcoma in HIV infected Patients. Acta Medica Marisiensis. 2016;62(2):285-288.
16. Myskowski PL, Niedzwiecki D, Shurgot BA, Kaufman D, Krown SE, Nisce L, Safai B. AIDS-associated Kaposi's sarcoma: variables associated with survival. J Am Acad Dermatol. 1988;18:1299-306.

17. Reid E, Suneja G, Ambinder RF, Ard K, Baiocchi R, Barta SK, et al. AIDS-related Kaposi sarcoma, version 2.2019. JNCCN Journal of the National Comprehensive Cancer Network. 2019 Feb;17(2):171-189.

18. Panel on Opportunistic Infections in Adults and Adolescents with HIV. Guidelines for the prevention and treatment of opportunistic infections in adults and adolescents with HIV: recommendations from the Centers for Disease Control and Prevention, the National Institutes of Health, and the HIV Medicine Association of the Infectious Diseases Society of America. Available at: http://aidsinfo.nih.gov/contentfiles/ Ivguidelines/adult_oi.pdf.

19. Vinhaes $C L$, Araujo-Pereira M, Tibúrcio R, Cubillos-Angulo JM, Demitto FO, Akrami KM, Andrade BB. Systemic Inflammation Associated with Immune Reconstitution Inflammatory Syndrome in Persons Living with HIV. Life (Basel). 2021;11(1):65.

20. Pinetta PP, Melendez J. Primary Lymph Node Kaposi Sarcoma in Two HIV postive patients presenting with generalized lymphadenopathy and pancytopenia in a third level hospital in Guatemala. Journal of AIDS and HIV treatment. 2019;1(1):13-18. 